\title{
Atom-number squeezing and bipartite entanglement of two-component Bose-Einstein condensates: analytical results
}

\author{
G. R. Jin *, X. W. Wang, D. Li, and Y. W. Lu \\ Department of Physics, Beijing Jiaotong University, Beijing 100044, China \\ E-mail: grjin@bjtu.edu.cn
}

\begin{abstract}
We investigate spin dynamics of a two-component Bose-Einstein condensates with weak Josephson coupling. Analytical expressions of atomnumber squeezing and bipartite entanglement are presented for atom-atom repulsive interactions. For attractive interactions, there is no number squeezing; however, the squeezing parameter is still useful to recognize the appearance of Schrödinger's cat state.
\end{abstract}

Keywords: Bose-Einstein condensates, Phase coherence, Number squeezing, Bipartite entanglement

PACS numbers: 03.75.Mn, 05.30.Jp, 42.50.Lc 
Atom-number squeezing and bipartite entanglement of two-component Bose-Einstein condensates: analytica

Atom-number squeezing has attracted much attention due to its potential applications in quantum metrology and quantum information [1, 2, 3, 4, 5, 6. As a special case of spin squeezed states [7, 8, 9, 10, 11, 12, 13, 14, 15, the number squeezed state shows reduced spin fluctuation of the $J_{z}$ component below the standard quantum limit (SQL), which in turn suppresses the deleterious effects arising from phase diffusion [16, 17, 18, 19, 20, 21]. Dynamical generation of the number squeezing has been theoretically investigated based upon a two-mode boson model with a weak Josephson coupling [22, 23, 25, 26, 27, 28].

So far, the number squeezing has been demonstrated indirectly by detecting an increased phase fluctuation [1, 6], but not atom-number variance. However, the variances of relative number and phase has a nontrivial relation so a direct measurement of the number fluctuation is of interests and necessary [27]. In this letter, we study atom-number squeezing and bipartite entanglement of a two-component BEC with the Josephson coupling. Two analytical expressions are discovered, which provides us a more direct way to measure the number variance and entropy of entanglement through extracting phase coherence (i.e., the visibility) in atomic interference experiments.

We consider a two-component Bose-Einstein condensate with hyperfine states $|1\rangle$ and $|2\rangle$ coupled by an external microwave (or rf) field [29, 30, 31, 32]. For the BEC confined in a deep three-dimensional harmonic potential, we adopt single-mode approximation [33, 34, 35, 36, which results in the following Hamiltonian $(\hbar=1)$ :

$$
H=\delta J_{z}-\Omega J_{x}+\chi J_{z}^{2},
$$

where the detuning $\delta$, the Josephson-like coupling constant $\Omega$, and the mean-field interaction strength $\chi$ can be controlled artificially. Angular momentum operators $J_{+}=\left(J_{-}\right)^{\dagger}=a_{2}^{\dagger} a_{1}$ and $J_{z}=\left(N_{2}-N_{1}\right) / 2$ are introduced, where $a_{\sigma}$ and $N_{\sigma}\left(=a_{\sigma}^{\dagger} a_{\sigma}\right)$ are the annihilation and number operators for the two modes $\sigma=1,2$. Eq. (1) is equivalent with a two-site Bose-Hubbard hamiltonian [37], where $\delta$ denotes potential bias of a double well, $\Omega$ the hopping term, and $\chi$ the on-site interaction. Total particle number $N=N_{1}+N_{2}$ is assumed to be a fixed c number, so atomic number operators $N_{\sigma}=j+(-1)^{\sigma} J_{z}$ with $\sigma=1,2$ and $j=N / 2$. Atom number fluctuations, defined as usual $\left(\Delta N_{\sigma}\right)^{2}=\left\langle N_{\sigma}^{2}\right\rangle-\left\langle N_{\sigma}\right\rangle^{2}$ are the same and equal to the variance $\left(\Delta J_{z}\right)^{2}$.

For any state vector $|\Psi\rangle$, one can determine the mean spin: $\langle\mathbf{J}\rangle=\left(\left\langle J_{x}\right\rangle,\left\langle J_{y}\right\rangle,\left\langle J_{z}\right\rangle\right)$, where $\left\langle J_{x}\right\rangle=\operatorname{Re}\left\langle J_{+}\right\rangle$and $\left\langle J_{y}\right\rangle=\operatorname{Im}\left\langle J_{+}\right\rangle$. The expectation value $\left\langle J_{+}\right\rangle$relates to the first-order cross correlation function [20, 21]:

$$
g^{(1)}=\frac{\left|\left\langle a_{2}^{\dagger} a_{1}\right\rangle\right|}{\sqrt{\left\langle N_{1}\right\rangle\left\langle N_{2}\right\rangle}}=\frac{\left|\left\langle J_{+}\right\rangle\right|}{\sqrt{j^{2}-\left\langle J_{z}\right\rangle^{2}}},
$$

which measures phase coherence of the two-component BEC. It is observable by extracting the visibility of atomic interference fringes [1, 2, 3, 4, 5, 6, 31, 37, 38. Similar definition of the phase coherence has been proposed in Refs. [39, 40]. The degree of atom-number squeezing is quantified by a parameter [4, 22,

$$
\xi^{2}=\frac{N\left(\Delta N_{\sigma}\right)^{2}}{\left\langle N_{1}\right\rangle\left\langle N_{2}\right\rangle}=\frac{2 j\left(\Delta J_{z}\right)^{2}}{j^{2}-\left\langle J_{z}\right\rangle^{2}},
$$


where total particle number $N=2 j$ is assumed to be fixed. It has been shown that there is no squeezing (i.e., $\xi^{2}=1$ ) for coherent spin state (CSS) [41]:

$$
|\theta, \phi\rangle=\exp \left[i \theta\left(J_{x} \sin \phi-J_{y} \cos \phi\right)\right]|j, j\rangle,
$$

which yields $\left\langle J_{z}\right\rangle=j \cos \theta$ and $\left(\Delta J_{z}\right)^{2}=(j / 2) \sin ^{2} \theta$. The condition of the number squeezing is therefore $\xi^{2}<1$ [22], which is consistent with previous one [6]: $\left(\Delta J_{z}\right)^{2}<j / 2$ for a symmetric BEC with population imbalance $\left\langle J_{z}\right\rangle=0$.

Firstly, let us consider spin dynamic of the symmetric BEC $(\delta=0)$ with repulsive interactions $(\chi>0)$ for an initial CSS state $|\Psi(0)\rangle=\left|\frac{\pi}{2}, 0\right\rangle=|j, j\rangle_{x}$, which is also an eigenvector of $J_{x}$ with eigenvalue $j$. Experimentally, such a state has been prepared by applying a two-photon $\pi / 2$ pulse to the condensed atoms occupied in the internal state $|2\rangle$ [29]. The state vector at any time $t$ can be expanded as $|\Psi\rangle=\sum_{m} c_{m}|j, m\rangle$, where the probability amplitudes $c_{m}$ are determined by time-dependent Schrödinger equation with the initial condition: $c_{m}(0)=\langle j, m \mid j, j\rangle_{x}=\frac{1}{2^{j}}\left(\begin{array}{c}2 j \\ j+m\end{array}\right)^{1 / 2}$. Note that the initial state shows the population imbalance $\left\langle J_{z}(0)\right\rangle=0$ and the number variance $\left(\Delta J_{z}(0)\right)^{2}=j / 2$ (i.e., $\xi^{2}=1$ ).

As the simplest case, we consider the Hamiltonian (1) with $\Omega=0$. This is the one-axis twisting model, proposed originally by Kitagawa and Ueda [7]. The phase coherence can be solved exactly as $g^{(1)}(t)=\cos ^{2 j-1}(\chi t)$. In the short-time limit, it decays exponentially as $g^{(1)}(t) \sim \exp \left[-\left(t / t_{d}\right)^{2}\right]$ with $\chi t_{d}=j^{-1 / 2}$, denoting a characteristic time scale for phase coherence [16, 17, 18, 19]. The damping of phase coherence, known as phase diffusion [16, 17, 18, 19, 20, 21] has been observed in experiment by extracting the visibility of the Ramsey fringe [38]. During phase diffusion, the number squeezing $\xi^{2}$ remains constant because of conserved probability distribution $\left|c_{m}\right|^{2}$.

The interplay between the nonlinear interaction and the Josephson coupling leads to suppressed phase diffusion due to the appearance of number squeezing. So far, the number squeezing has been demonstrated for the BEC in optical lattices [1, 2, 3, 4], optical trap [5], and atom chip [6]. To understand how this works, we consider unitary evolution of the symmetric BEC governed by Hamiltonian (11). In Schrödinger picture, the amplitudes always satisfy the relation $c_{-m}=c_{m}$, which in turn leads to $\left\langle J_{y}\right\rangle=\left\langle J_{z}\right\rangle=0$ and $\left\langle J_{x}\right\rangle=\left\langle J_{+}\right\rangle \neq 0$. Moreover, Eq. (2) and Eq. (3) now reduce to $g^{(1)}=\left|\left\langle J_{x}\right\rangle\right| / j$ and $\xi^{2}=2\left\langle J_{z}^{2}\right\rangle / j$. A relation between $g^{(1)}$ and $\xi^{2}$ can be obtained by examining Heisenberg equations of motion:

$$
\begin{aligned}
& \dot{J}_{x}=-\chi\left(J_{y} J_{z}+J_{z} J_{y}\right), \\
& \dot{J}_{y}=\Omega J_{z}+\chi\left(J_{x} J_{z}+J_{z} J_{x}\right), \\
& \dot{J}_{z}=-\Omega J_{y} .
\end{aligned}
$$

For time-independent $\Omega$ and $\chi$, we have $d J_{z}^{2} / d t=(\Omega / \chi) d J_{x} / d t$, and thus

$$
\xi^{2}(t)=1-\frac{2 \Omega}{\chi}\left[1 \mp g^{(1)}(t)\right]
$$

with the upper sign for the mean spin $\left\langle J_{x}\right\rangle \geq 0$ and the lower one for $\left\langle J_{x}\right\rangle<0$. Eq. (8) provides us an exact relation between the number squeezing $\xi^{2}$ and the 
Atom-number squeezing and bipartite entanglement of two-component Bose-Einstein condensates: analytica
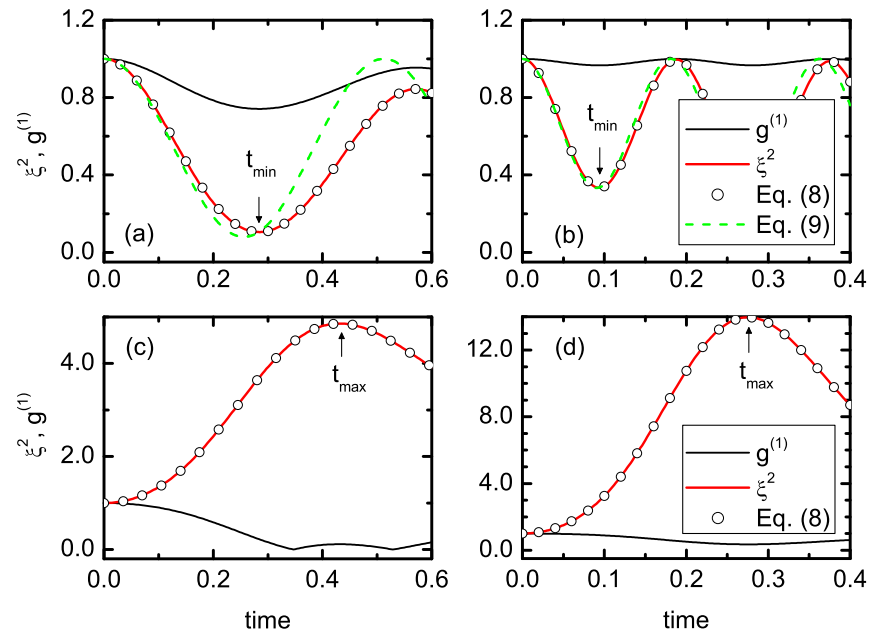

Figure 1. (Color online) Time evolution of phase coherence $g^{(1)}$ (black thin) and number-squeezing parameter $\xi^{2}$ (red) for $N=20$, and the coupling: $\Omega /|\chi|=1.732$ (a) and (c), $\Omega /|\chi|=10$ (b) and (d). In (c) and (d), negative $\chi$ case is considered. The circles and the dashed green line are given by Eq. (8) and Eq. (91), respectively. The time is in units of $|\chi|^{-1}$.

phase coherence $g^{(1)}$. To confirm it, we consider two-particle $(N=2)$ case. It is easy to obtain $g^{(1)}=1-\frac{1}{2}\left(\chi \sin \omega_{2} t / \omega_{2}\right)^{2}$ and $\xi^{2}=1-\Omega \chi\left(\sin \omega_{2} t / \omega_{2}\right)^{2}$, with $\omega_{2}=\left(\Omega^{2}+\chi^{2} / 4\right)^{1 / 2}$. Obviously, Eq.(8) holds for the two-particle case. In addition, we find that local minimum of $\xi^{2}$ (i.e., maximal number squeezing) occurs at time $t_{\min }=\pi /\left(2 \omega_{2}\right)$. If an optimal coupling $\Omega / \chi=1 / 2$ is applied, the system will evolve into maximally number-squeezed state (MSS): $|\Psi\rangle_{\mathrm{MSS}}=i e^{-i \pi /(2 \sqrt{2})}|1,0\rangle$, which exhibits perfect squeezing $\left(\Delta J_{z}\right)^{2}=0$ [8].

For large $N$ case, there exist no exact solutions; however, some approximated solutions are obtainable if the coupling is strong enough. In this case, the mean spin $\left\langle J_{x}\right\rangle$ almost remains unchanged at $j$. As a result, we adopt frozen-spin approximation (FSA) [8, 23, 24, i.e., replacing $J_{x}$ by $j(=N / 2)$ in the Heisenberg equations, and obtain $\ddot{J}_{z}=-\Omega^{2} J_{z}-\Omega \chi\left(J_{x} J_{z}+J_{z} J_{x}\right) \simeq-\omega_{N}^{2} J_{z}$, where $\omega_{N}=\sqrt{\Omega(\Omega+N \chi)}$ [23]. Solving the above equation, it is easy to get harmonic solutions of $J_{y}(t)$ and $J_{z}(t)$ 24]. Inserting them into Eq. (5), we obtain $J_{x}(t)$ and also $g^{(1)}=\left|\left\langle J_{x}\right\rangle\right| / j \simeq 1-\frac{N}{2}\left(\chi \sin \omega_{N} t / \omega_{N}\right)^{2}$. Now, the phase coherence $g^{(1)}(t)$ almost exhibits sinusoidal oscillations [thin lines of Fig. 1, but not exponential damping as previous case. In other words, the phase diffusion is strongly suppressed due to the Josephson coupling. From Eq. (8), we further obtain

$$
\xi^{2}(t) \simeq 1-N \Omega \chi\left(\frac{\sin \omega_{N} t}{\omega_{N}}\right)^{2} .
$$

Clearly, the maximal squeezing appears at time $t_{\min }=\pi /\left(2 \omega_{N}\right)$ with, $\xi_{\min }^{2}=\Omega^{2} / \omega_{N}^{2}<1$ [23, 24]. Dunningham et al. 25] has independently derived the time $t_{\min }$ using a semiclassical analysis of Hamiltonian (11) 34. For large $N$ case $\left(>10^{3}\right)$, the optimal coupling obeys the power rule $\Omega / \chi \sim 0.58 N^{0.32}$ [26, 27]. In Fig. 1](a), time evolution 
Atom-number squeezing and bipartite entanglement of two-component Bose-Einstein condensates: analytica

of $g^{(1)}$ and $\xi^{2}$ is plotted for the optimal coupling $\Omega / \chi=1.732$ and $N=20$. The FSA works (green dashed curves) quite well to predict $t_{\min }$. For relatively large $\Omega / \chi$, say $\Omega / \chi=10$ for $N=20$, the FSA follows full evolution of $\xi^{2}$ [see Fig. 1 (b)]. As shown Fig. 1(c) and (d), Eq.(8) keeps hold for negative $\chi$ case [see below].

The relation between spin squeezing and quantum entanglement is of interests. It was shown that the obtained squeezing is useful for quantum metrology 8, 9] and entanglement [12], provided that $\zeta_{\mathrm{S}}=\sqrt{2 j}\left(\Delta \hat{J}_{z}\right) /\left|\left\langle\hat{J}_{+}\right\rangle\right|=\xi / g^{(1)}<1$ [4]. For the system considered here, however, only entanglement between the two modes (i.e., bipartite entanglement) is accessible due to the indistinguishability of identical bosons [43]. A standard measure of bipartite entanglement is the so-called entropy of entanglement [43, 44]:

$$
E(t)=-\operatorname{Tr}\left[\rho_{1} \log \left(\rho_{1}\right)\right]=-\sum_{m=-j}^{j}\left|c_{m}\right|^{2} \log \left(\left|c_{m}\right|^{2}\right),
$$

where $\rho_{1}=\operatorname{Tr}_{2}(\rho)$ is the reduced density operator for mode 1 obtained by partial trace over mode 2. The value of $E$ varies between 0 , for the separable product states to a maximum of $\log (d)$, for maximally entangled states $|\Psi\rangle_{\text {MES }}=d^{-1 / 2} \sum_{m}|j, m\rangle$, where $d=(2 j+1)$ is the dimension of the Hilbert space. Utilizing $c_{m}=d^{-1 / 2}$, Eq. (10) gives $E_{\max }=\log (d)$, which is the maximum value of the entanglement for the system [43, 44]. As an ansanz, the spin state $|\Psi(t)\rangle$ can be treated as a Guassian [17]:

$$
\left|c_{m}\right|^{2} \simeq \frac{1}{\left[2 \pi\left(\Delta J_{z}\right)^{2}\right]^{1 / 2}} \exp \left[-\frac{m^{2}}{2\left(\Delta J_{z}\right)^{2}}\right]
$$

with its width $\left(\Delta J_{z}\right)=(j / 2)^{1 / 2} \xi$, determined by the number squeezing parameter $\xi^{2}(t)$. Substituting Eq. (11) into Eq. (10), and replacing the discrete sum over $m$ by an integral, we arrive at

$$
E(t) \simeq \frac{1}{2} \log \left[e \pi j \xi^{2}(t)\right]
$$

which provides us analytical relation between the number squeezing and the two-mode entanglement. Considering $\xi^{2}=1$ for the initial CSS $|j, j\rangle_{x}$, we have $E(0)=E_{\mathrm{CSS}} \simeq$ $\frac{1}{2} \log [e \pi j]$. Numerically, Hines et al. [43] have found that $E_{\mathrm{CSS}} / E_{\max }$ remains finite for large $N$. Our result shows $E_{\mathrm{CSS}} / E_{\max } \rightarrow \frac{1}{2}$ as $N \rightarrow \infty$. In Fig. 2(a) and (b), we plot time evolution of the entropy for finite $N=20$ case. One can find that Eq. (12) (red circles) agrees very well with exact numerical solution of Eq. (10). In the initial stage, $E(t)$ decreases from $E_{\mathrm{CSS}}$ to its local minimum at $t_{\min }$, due to the appearance of the MSS. In comparison with the CSS, the MSS approaches to localized Twin-Fock state [45]: $|N / 2\rangle_{1}|N / 2\rangle_{2}=|j, 0\rangle$, which exhibits $g^{(1)}=\xi^{2}=E=0$. To confirm it, we plot evolution of probability distribution $\left|c_{m}\right|^{2}$ in Fig. 3(a). For the optimal coupling $\Omega / \chi=1.732$ and $N=20$, the system evolves into the MSS after a duration $\chi t_{\min }=0.284$, which shows probability distribution peaked at $m=0$. Quasi-probability distribution $Q(\theta, \phi)$ of the initial state is isotropic [Fig. 3(c)], representing the minimal uncertainty relationship of a coherent state [7]. It becomes an elliptic shape due to the squeezing along $J_{z}$ and the anti-squeezing along $J_{y}$ [Fig. [3 (d)]. 
Atom-number squeezing and bipartite entanglement of two-component Bose-Einstein condensates: analytica
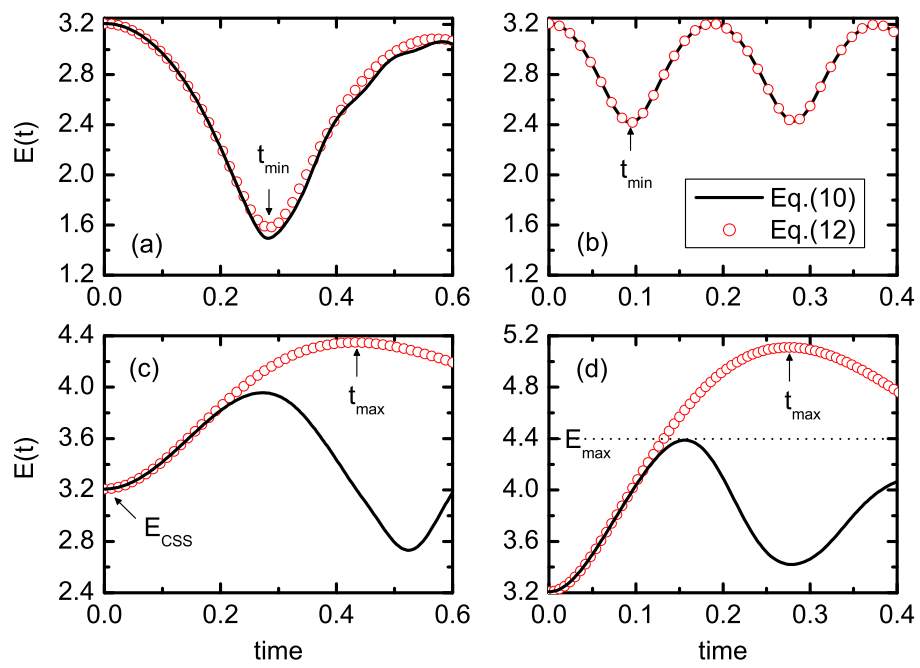

Figure 2. (color online) The entropy of entanglement $E$ (black solid lines) for $N=20$. The red circles are given by Eq. (12) with numerical result of $\xi^{2}$. The initial value of $E, E_{\mathrm{CSS}}=\frac{1}{2} \log [e \pi N / 2]=3.21$, and the maximal value $E_{\max }=\log (N+1)=4.39$. Other parameters are the same with Fig. [1.

Until now, we consider spin dynamics of Hamiltonian (1) with $\chi>0$ and the initial state $|j, j\rangle_{x}$. This state is a stable fixed point in phase space [42, so the phase coherence $g^{(1)}$, the number squeezing $\xi^{2}$, and the two-mode entanglement $E$ oscillate regularly with the same period. Using Eq. (8) and Eq. (12), it is possible to measure both $\xi^{2}$ and $E$ by extracting $g^{(1)}$ (i.e., the visibility) in atomic interference experiment [1, 2, 3, 4, 5, 6, 31, 38]. There are two alternative experimental setups. One possibility is to trap the condensed ${ }^{23} \mathrm{Na}$ atoms in a symmetric double-well potential formed by atom chip [6], the other is the two-component BEC in an optical dipole trap [30]. In both cases, positive and large enough $\chi \sim\left(a_{11}+a_{22}-2 a_{12}\right)$ is required, which speeds up dynamics of the system such that the deleterious effects like atom losses can be neglected [25].

To proceed, let us consider another scenario: spin dynamics of Hamiltonian (1) with negative $\chi$ case [46]. Now, the initial state $|j, j\rangle_{x}$ corresponds to an unstable point at the separatrix [42], which leads to a more complex dynamics with a quite different characteristic time scale. From Eq. (8), we find that there is no number squeezing for negative $\chi$ case [see also Fig. 1(c) and (d)]. Instead of preparing the MSS, the latter model can be used to generate Schrödinger's cat state likes, $|\Psi\rangle_{\mathrm{CAT}}=\frac{1}{\sqrt{2}}(|j,-j\rangle+|j, j\rangle)$ [47. In Fig. 2(c) and (d), we plot time evolution of $E(t)$ for $N=20$ case. Our results show that Eq. (12) follows exact results of $E$ in the early stages of the evolution, then

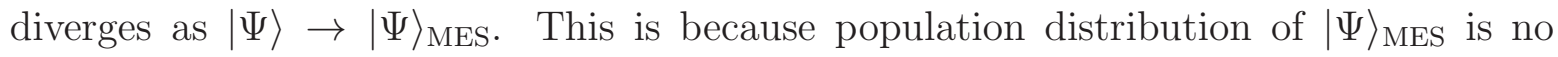
longer to be a Gaussian, and Eq. (12) can not simulate the entropy. However, right-side of Eq. (12), a monotonic function of $\xi^{2}$, is still useful to recognize the appearances of the cat state, which exhibits the largest number variance $\left(\Delta J_{z}\right)_{\max }^{2}=j^{2}$ and $\xi_{\max }^{2}=2 j$. In real evolution, an approximate cat state with $\xi^{2} \sim 1.3 j$ [Fig. [(d)] can be obtained 
Atom-number squeezing and bipartite entanglement of two-component Bose-Einstein condensates: analytica at a time $|\chi| t_{\max } \sim \ln (8 N) / N$ for the optimal coupling $\Omega /|\chi|=N / 2$ [47]. From right panel of Fig. 3, we also find that this state shows probability distribution $\left|c_{m}\right|^{2}$ peaked at $m= \pm j$ and maximal values of $Q(\theta, \phi)$ pointed to the north and the south poles of the Bloch sphere.

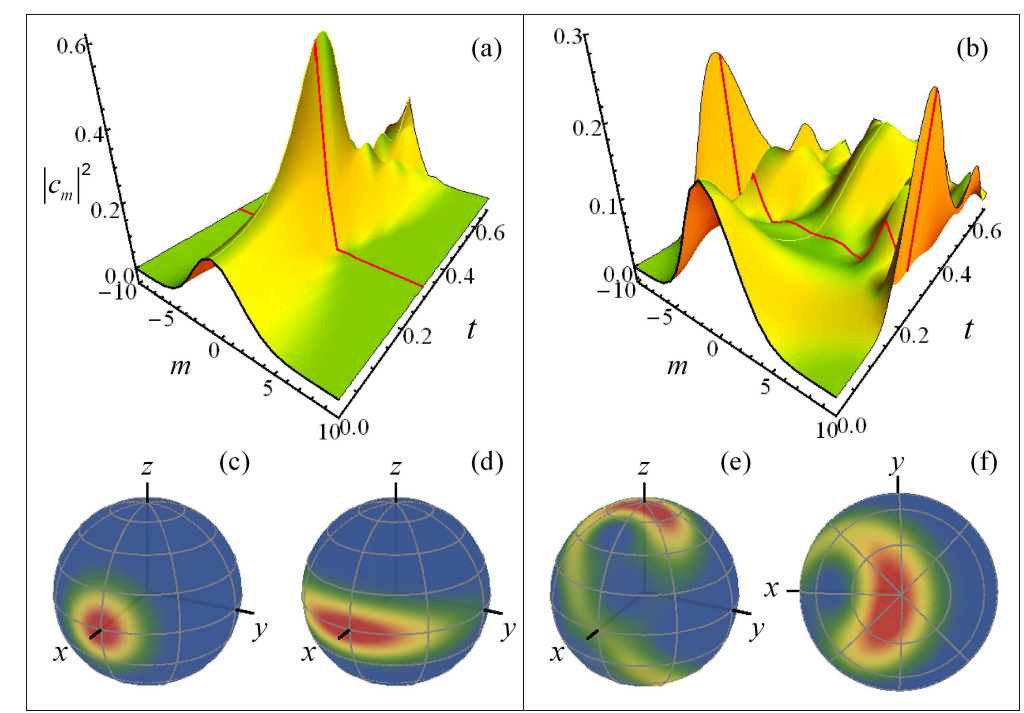

Figure 3. (color online) Probability distribution $\left|c_{m}\right|^{2}$ (a)-(b), and quasi-probability distribution $Q(\theta, \phi)=|\langle\theta, \phi \mid \Psi(t)\rangle|^{2}$ (c)-(f), where the CSS $|\theta, \phi\rangle$ is given in Eq. (4). The red lines in (a) and (b) represents $\left|c_{m}\right|^{2}$ for the MSS at $t_{\min }=0.284 \chi^{-1}$ and the cat state at $t_{\max }=0.275|\chi|^{-1}$, respectively. The parameters in the left and the right panels are the same with Fig. प(a) and Fig. 1(d).

In summary, we have investigated spin dynamics of a symmetric BEC with repulsive interactions $(\chi>0)$ evolved from a coherent spin state $|j, j\rangle_{x}$. As main results of our work, we find analytical expressions of the number-squeezing parameter $\xi^{2}$ and the entropy of entanglement $E$ as, Eq. (8) and Eq. (12). Both of them can be, in principle, at least, measured by extracting the phase coherence $g^{(1)}(t)$ (i.e., the visibility) in atomic interference experiments. For the case of attractive interactions $(\chi<0)$, thought there exists no number squeezing, the squeezing parameter $\xi^{2}$ or Eq. (12) is still useful to recognize the appearance of Schrödinger's cat state.

\section{Acknowledgments}

This work is supported by the NSFC (Contract No. 10804007), the SRFDP (Contract No. 200800041003), and Research Funds of Beijing Jiaotong University (Grants No. 2007XM049).

\section{References}

[1] Orzel O et al 2001 Science 2912386

Li W, Tuchman A K, Chien H-C, and Kasevich M A 2007 Phys. Rev. Lett. 98040402 
Atom-number squeezing and bipartite entanglement of two-component Bose-Einstein condensates: analytica

[2] Gerbier F et al 2006 Phys. Rev. Lett. 96090401

Greiner M et al 2002 Nature $4 \mathbf{1 5} 39$

Jaksch D et al 1998 Phys. Rev. Lett. 813108

[3] Sebby-Strabley J et al 2007 Phys. Rev. Lett. 98 (2007) 200405

[4] Esteve J et al 2008 Nature $\mathbf{4 5 5} 1216$

[5] Chuu C-S et al 2005 Phys. Rev. Lett. 95260403

[6] Jo G-B et al 2007 Phys. Rev. Lett. 98030407

[7] Kitagawa M and Ueda M 1993 Phys. Rev. A 475138

[8] Wineland D J et al 1994 Phys. Rev. A 5067 Wineland D J et al, Phys. Rev. A 199246 R6797

[9] Meyer V et al 2001 Phys. Rev. Lett. 865870 Leibfried D et al 2004 Science 3041476

[10] Hald J, Sørensen J L, Schori C, and Polzik E S 1999 Phys. Rev. Lett. 831319

[11] Kuzmich A, Mandel L, and Bigelow N P 2000 Phys. Rev. Lett. 851594

[12] Sørensen A et al 2001, Nature 40963

[13] Wang X and Sanders B C 2003 Phys. Rev. A 68012101

[14] Geremia J M et al 2004 Science 304270

[15] Jin G R, Liu Y C, and Liu W M 2009 New J. Phys. 11073049

[16] Wright E M, Walls D F, and Garrison J C 1996 Phys. Rev. Lett. 772158

[17] Imamoḡlu A, Lewenstein M, and You L 1997 Phys. Rev. Lett. 782511

[18] Javanainen J and Wilkens M 1997 Phys. Rev. Lett. 784675

[19] Castin Y and Dalibard J 1997 Phys. Rev. A 554330

[20] Law C K, Pu H, Bigelow N P, and Eberly J H 1998 Phys. Rev. A 58531

[21] Khodorkovsky Y, Kurizki G, and Vardi A 2008 Phys. Rev. Lett. 100220403

[22] Raghavan S, Pu H, Meystre P, and Bigelow N P 2001 Opt. Commu. 188149

[23] Law C K, Ng H T, and Leung P T 2001 Phys. Rev. A 63055601

[24] Bhattacherjee A, Ranjan V, and Mohan M 2003 Inter. J. Mod. Phys. B 172579

[25] Dunningham J A, Burnett K, and Edwards M 2001 Phys. Rev. A 64015601

[26] Jin G R and Kim S W 2007 Phys. Rev. Lett. 99170405 Jin G R and Kim S W 2007 Phys. Rev. A 76043621

[27] Jin G R and Law C K 2008 Phys. Rev. A 78063620

[28] Grond J, Schmiedmayer J, and Hohenester U 2009 Phys. Rev. A 79 021603(R)

[29] Hall D S et al 1998 Phys. Rev. Lett. 811539

[30] Stenger J et al 1998 Nature 396345

[31] Schumm T et al 2005 Nature Phys. 157 Shin Y et al 2005 Phys. Rev. A 72 021604(R)

[32] Anderson R P et al 2009 Phys. Rev. A 80023603 Hall B V et al 2007, Phys. Rev. Lett. 98030402.

[33] Milburn G J, Corney J, Wright E M, and Walls D F 1997 Phys. Rev. A 554318

[34] Smerzi A et al 1997 Phys. Rev. Lett. 794950 Raghavan S et al 1999 Phys. Rev. A 59620

[35] Cirac J I, Lewenstein M, Mølmer K, and Zoller P 1998 Phys. Rev. A 571208

[36] Vardi A and Anglin J R 2001 Phys. Rev. Lett. 86568

[37] Fölling S et al 2007 Nature 4481029

[38] Widera A et al 2008 Phys. Rev. Lett. 100140401

[39] Jääskelänen M, Zhang W, and Meystre P 2004 Phys. Rev. A 70063614 Jääskelänen M and Meystre P 2005 Phys. Rev. A 71043603

[40] Lee C, Fu L B, and Kivshar Y S 2008 Eur. Phys. Lett. 8160006

[41] Radcliffe J M 1971 J. Phys. A 4313 Arecchi F T et al 1972 Phys. Rev. A 62211

[42] Boukobza E, Chuchem M, Cohen D, and Vardi A 2009 Phys. Rev. Lett. 102180403 
Atom-number squeezing and bipartite entanglement of two-component Bose-Einstein condensates: analytica

[43] Hines A P, McKenzie R H, Milburn G J 2003 Phys. Rev. A 67013609

[44] Nielsen M A and Chuang I L 2000, Quantum computation and quantum information (Cambridge: Cambridge University Press)

[45] Holland M J, Burnett K 1993 Phys. Rev. Lett. 711355

Bouyer P and Kasevich M A 1997 Phys. Rev. A 56 R1083

Rodríguez R et al 2007 Phys. Rev. A 75 011601(R)

[46] Phase seperation of the two-component BEC occurs for negative $\chi$ case, which can be avoided by considering positive $\chi$ and the intial state $|j,-j\rangle_{x}$ (cf. Ref. 43). Our results still hold.

[47] Micheli A, Jaksch D, Cirac J I, and Zoller P 2003 Phys. Rev. A 67, 013607 